\title{
Zeitschrift für Gerontologie und Geriatrie
}

\section{Patient-reported outcome and experience measures in geriatric emergency medicine --Manuscript Draft--}

Manuscript Number:

Full Title:

Article Type:

Keywords:

Corresponding Author:
ZFGG-D-20-00093R1

Patient-reported outcome and experience measures in geriatric emergency medicine

Themenschwerpunkt

Emergency medicine; geriatrics; Frailty; patient-reported outcome measures; person-centred

James David van Oppen

University of Leicester

Leicester, Leicestershire UNITED KINGDOM

Corresponding Author Secondary Information:

Corresponding Author's Institution:

University of Leicester

Corresponding Author's Secondary Institution:

First Author:

First Author Secondary Information:

Order of Authors:

James D van Oppen

Jose M Valderas

Nicola J Mackintosh

Simon P Conroy

\section{Order of Authors Secondary Information:}

Funding Information:

Abstract:

Response to Reviewers:
James D van Oppen

Research Trainees Coordinating Centre Dr James D van Oppen (Academic Clinical Fellowship)

Older people with frailty and health crises have complex physical and social needs. Modern emergency care systems are fast-flowing, using protocols optimised for singleproblem presentations. Systems must incorporate individualised care in order to bestserve people with multiple problems.

Healthcare quality is typically appraised with service metrics such as department length of stay and mortality. Worldwide, Patient-Reported Outcome Measures (PROMs) and Experience Measures (PREMs) are increasingly used in research, service development and performance evaluation, paving the ground for their use to support individual clinical decision-making. PROMs and PREMs are person-centred metrics which at individual level inform healthcare decisions and which at strategic level drive improvement through inter-provider effectiveness comparison. To date, there is no PROM or PREM specifically developed for older people with frailty and emergency care needs.

Thank you the reviewers for their comments on our article. In our revised manuscript, we have made the necessary corrections to our references. 


\section{Patient-reported outcome and experience measures in geriatric emergency medicine}

van Oppen, James $\mathrm{D}^{1,2}$

Valderas, Jose $\mathrm{M}^{3}$

Mackintosh, Nicola $\mathrm{J}^{1}$

Conroy, Simon P ${ }^{1,2}$

${ }^{1}$ Department of Health Sciences, University of Leicester, LE1 7RH, UK

${ }^{2}$ Emergency and Specialist Medicine, University Hospitals Leicester NHS Trust, LE1 5WW, UK

${ }^{3}$ Health Services and Policy Research Group, University of Exeter, EX1 2LU, UK

jvo3@leicester.ac.uk

\section{Abstract}

Older people with frailty and health crises have complex physical and social needs. Modern emergency care systems are fast-flowing, using protocols optimised for single-problem presentations. Systems must incorporate individualised care in order to best-serve people with multiple problems.

Healthcare quality is typically appraised with service metrics such as department length of stay and mortality. Worldwide, Patient-Reported Outcome Measures (PROMs) and Experience Measures (PREMs) are increasingly used in research, service development and performance evaluation, paving the ground for their use to support individual clinical decision-making. PROMs and PREMs are person-centred metrics which at individual level inform healthcare decisions and which at strategic level drive improvement through interprovider effectiveness comparison. To date, there is no PROM or PREM specifically developed for older people with frailty and emergency care needs. 


\title{
Patient-reported outcome and experience measures in geriatric emergency medicine
}

\begin{abstract}
Older people with frailty and health crises have complex physical and social needs. Modern emergency care systems are fast-flowing, using protocols optimised for single-problem presentations. Systems must incorporate individualised care in order to best-serve people with multiple problems.
\end{abstract}

Healthcare quality is typically appraised with service metrics such as department length of stay and mortality. Worldwide, Patient-Reported Outcome Measures (PROMs) and Experience Measures (PREMs) are increasingly used in research, service development and performance evaluation, paving the ground for their use to support individual clinical decision-making. PROMs and PREMs are person-centred metrics which at individual level inform healthcare decisions and which at strategic level drive improvement through interprovider effectiveness comparison. To date, there is no PROM or PREM specifically developed for older people with frailty and emergency care needs.

\section{Introduction}

Older people living with frailty have poorer health outcomes after even short hospital stays [15]. Emergency Departments (ED) in most countries have observed annual increases in attendances; around one fifth of people attending German departments are aged over 80 [26]. In the UK, approximately three million annual ED attendances are by people living with frailty. Co-ordinated efforts are underway to improve healthcare outcomes for older people with frailty and emergency care needs, including through specialised training and focussed research [9].

Traditional fast-flowing, protocol-driven emergency care systems are well-suited for people with single problems, but may underserve people with atypical presentations or complex co-morbidities [2, 21]. Systems tend to require multi-disciplinary, holistic, person-centred care in order to best-serve people living with frailty [19]. The geriatric emergency medicine sections of the European Society for Emergency Medicine (EUSEM) and the European Union Geriatric Medicine Society (EUGMS) have a shared objective to inform service delivery programmes and research agendas in order to tailor current emergency medicine services for this group [11].

Quality of emergency care for older people is typically reported using service metrics such as length of stay and readmission rate [5]. However, people living with frailty consider additional metrics related to health outcomes and preferences for care to also be important and meaningful. Accordingly, the degree to which the provision of care supports patients in 
reaching their goals as defined around these outcomes and preferences is important for capturing high quality care.

\section{Outcomes and preferences for healthcare}

Older people with frailty have additional, and perhaps unique needs when they attend the ED $[21,26]$. Patient needs can be defined in terms of selected health outcomes or aspects of health status. For older people living with frailty these outcomes include symptoms (mood, pain), functional status (autonomy, activities of daily living, loneliness), survival, quality of life, and carer burden $[1,30]$.

Individuals' preferences vary for specific processes and outcomes of health care [18]. Healthcare preferences incorporate the processes or procedures someone is willing to undergo in order to achieve their health outcome goals. Experience of these processes can be determined by individualised care, clinical communication, emotional and personal care, and the physical environment $[12,24]$. In the emergency care context, adults of all age groups consider prompt waiting time, understandable information, and getting a diagnosis to be important aspects of experience [10]. People's experience of processes within a healthcare system are measurable using Patient-Reported Experience Measures (PREMs), usually in the form of questionnaires [7].

Many outcomes relevant to frail patients, including pain, daily function, and quality of life are not available in current automated quality metrics, but are measurable using PatientReported Outcome Measures (PROMs). PROMs are questionnaires that capture people's perceptions of their current health state and of their overall health outcomes. Personcentred measurement of health outcomes and experiences can be simultaneous using a combined instrument. The instruments can therefore measure care effectiveness and contribute to its improvement.

\section{Applications of person-reported outcomes and experience measures}

Patient-reported outcomes are increasingly recognised as valid approaches to measure the quality and impact of care by clinical practitioners and those involved in health service administration, planning and purchasing. When the data is considered at the 'macro' level on a wide scale [23], PROM and PREM metrics stimulate service improvement and redesign initiatives through measurement and comparison before, during, and after healthcare interventions [4, 25]. PROMs and PREMs are used routinely in trial research and some clinical settings [17], for instance to measure treatment effectiveness in elective hip surgery and asthma.

At the 'micro' level - the clinician-patient interface - routine use of these instruments has been demonstrated to improve process and outcomes of care. Use of PROM instruments can afford people the freedom to reflect on their health conditions and priorities and can enable self-management to some extent $[3,29]$. Use of PROMs during clinical consultations prompts patients and clinicians to raise and discuss issues, and thereby can facilitate communication of those important perspectives and health outcome goals by informing an agenda for clinical conversations [14]. Elicitation of individuals' perspectives and health outcome goals reinforces perceptions of self-efficacy and improves patient activation [22]. These concepts are related to individuals' confidence and ability to manage their health 
state. Self-efficacy and activation are measurable and are independently associated with better health outcomes [13].

Following over two decades of interest in person-centred outcome measurement among psychometric academics, there has been a slow but progressive translation of instruments into routine clinical practice. The impact of PROMs and PREMs on patients themselves has been studied in systematic reviews and randomised controlled trials. Identifying mechanisms to best feedback PROM and PREM data to clinicians is a topic of ongoing enquiry [22]. The feasibility and impact of PROM and PREM implementation in emergency care settings, particularly with people who are frail, have not yet been investigated in depth.

\section{PROMs and PREMs for older people with frailty and emergency care needs}

There is no validated PROM or PREM specific for older people with frailty and emergency care needs. Instrument questions are developed and tested for validity to evidence-based domains of care $[6,8]$. Responses are typically scaled. Analysis could produce not only a numerical score for research and quality improvement applications, but also an individualised overview of the user's health outcome goals.

Enabling individualised, person-centred geriatric emergency care through empowered communication might improve that currently delivered through existing single-problem pathways. Clinicians could offer better person-centred healthcare if armed earlier with greater awareness of their patients' preferences and priorities [20]. Availability of data at the time people access ED care could inform clinical conversations and enhance shared decision-making. Data could be rapidly abstracted and presented at the clinician-patient interface if collected electronically. Older people have been shown to find electronic instruments acceptable provided their accessibility needs have been considered during development [16]. Clinicians could be further empowered with knowledge of people's perspectives through users' supported communication using individualised instruments [28] such as the Patient-Generated Index [27].

There is clear potential benefit from the early routine collection of PROMs and PREMs in relation to emergency care episodes. However, the ED environment poses an inherent challenge due to the rapid pace and people's unwell health states. Investigation is required to determine the feasibility of data collection in emergency care settings. While older people living with frailty may have greater potential benefit from the earlier individualised care enabled with person-centred measures, research and innovation is required to overcome communication, cognitive, and sensory barriers which they frequently live with. The effectiveness and impact of PROMs applied as communication support tools is as yet unproven in this population but has the potential to inspire a step change in how care is designed and delivered.

\section{Compliance with ethics guidelines}

Ethical approval was not required for this topic review. 


\section{Conflicts of interest}

The authors have no conflicts of interest to declare.

\section{References}

1. Akpan A, Roberts C, Bandeen-Roche K et al. (2018) Standard set of health outcome measures for older persons. BMC Geriatr 18:36

2. Banerjee J, Conroy S, Cooke MW (2013) Quality care for older people with urgent and emergency care needs in UK emergency departments. Emerg Med J 30:699-700

3. Black N (2013) Patient reported outcome measures could help transform healthcare. BMJ 346:f167

4. Black N, Varaganum M, Hutchings A (2014) Relationship between patient reported experience (PREMs) and patient reported outcomes (PROMs) in elective surgery. BMJ Qual Saf 23:534-542

5. Burkett E, Martin-Khan M, Gray L (2017) Quality indicators in the care of older persons in the emergency department: A systematic review of the literature. Australas J Ageing 36:286-298

6. Butt Z, Reeve B (2012) Enhancing the patient's voice: Standards in the design and selection of patient-reported outcomes measures (PROMs) for use in patientcentered outcomes research. Contracted report for the Patient-Centered Outcomes Research Institute (PCORI). http://www.pcori.org/assets/Enhancing-the-PatientsVoice-Standards-in-the-Design-and-Selection-of-Patient-Reported-OutcomesMeasures-for-Use-in-Patient-Centered-Outcomes-Research.pdf. Accessed on 01/07/2020

7. Calvert M, Kyte D, Price $G$ et al. (2019) Maximising the impact of patient reported outcome assessment for patients and society. BMJ 364:k5267

8. Cappelleri JC, Zou KH, Bushmakin AG et al. (2014) Patient-reported outcomes: measurement, implementation and interpretation. CRC Press/Taylor \& Francis, Boca Raton, $\mathrm{FL}$

9. Conroy S, Nickel CH, Jónsdóttir AB et al. (2016) The development of a European curriculum in Geriatric Emergency Medicine. Eur Geriatr Med 7:315-321

10. Curran J, Cassidy C, Chiasson D et al. (2017) Patient and caregiver expectations of emergency department care: A scoping literature review. Int Emerg Nurs 32:62-69

11. EUSEM (2018) Section of Geriatric Emergency Medicine. https://eusem.org/sectionsand-committees/sections/geriatric-section. Accessed on 01/07/2020

12. Graham B, Endacott R, Smith JE et al. (2019) 'They do not care how much you know until they know how much you care': a qualitative meta-synthesis of patient experience in the emergency department. Emerg Med J 36:355-363

13. Greene J, Hibbard JH (2012) Why does patient activation matter? An examination of the relationships between patient activation and health-related outcomes. J Gen Intern Med 27:520-526

14. Greenhalgh J, Gooding K, Gibbons E et al. (2018) How do patient reported outcome measures (PROMs) support clinician-patient communication and patient care? A realist synthesis. J Patient Rep Outcomes 2:42

15. Keeble E, Roberts HC, Williams CD et al. (2019) Outcomes of hospital admissions among frail older people: a 2-year cohort study. Br J Gen Pract 69:e555-e560 
16. Mannheim I, Schwartz E, Xi W et al. (2019) Inclusion of plder adults in the research and design of digital technology. Int J Environ Res Public Health 16:3718

17. Mason N, Sox H, Whitlock E (2019) A patient-centered approach to comparative effectiveness research focused on older adults: Lessons from the patient-centered outcomes research institute. J Am Geriatr Soc 67:21-28

18. Naik AD, Dindo LN, Van Liew JR et al. (2018) Development of a clinically feasible process for identifying individual health priorities. J Am Geriatr Soc 66:1872-1879

19. NICE (2012) CG138: Patient experience in adult NHS services: improving the experience of care for people using adult NHS services.

https://www.nice.org.uk/guidance/cg138/chapter/1-guidance. Accessed on 25/09/2018

20. Parke B, Hunter K, Schulz M et al. (2016) Know me - A new person-centered approach for dementia-friendly emergency department care. Dementia 18:432-447

21. Perry A, Tejada JM, Melady D (2018) An approach to the older patient in the emergency department. Clin Geriatr Med 34:299-311

22. Porter I, Gonçalves-Bradley D, Ricci-Cabello I et al. (2016) Framework and guidance for implementing patient-reported outcomes in clinical practice: evidence, challenges and opportunities. J Comp Eff Res 5:507-519

23. Schick-Makaroff K, Karimi-Dehkordi M, Cuthbertson L et al. (2020) Using patient- and family-reported outcome and experience measures across transitions of care for frail older adults living at home: A meta-narrative synthesis. Gerontologist:gnz162 [Epub ahead of print]

24. Shankar KN, Bhatia BK, Schuur JD (2014) Toward patient-centered care: a systematic review of older adults' views of quality emergency care. Ann Emerg Med 63:529550.e521

25. Singh I, Aithal S (2018) Selecting best-suited "patient-related outcomes" in older people admitted to an acute geriatric or emergency frailty unit and applying quality improvement research to improve patient care. Patient Relat Outcome Meas 9:309320

26. Singler K, Christ M, Sieber C et al. (2011) Geriatrische Patienten in Notaufnahme und Intensivmedizin. Der Internist 52:934-938

27. Tang JA, Oh T, Scheer JK et al. (2014) The current trend of administering a patientgenerated index in the oncological setting: a systematic review. Oncol Rev 8:245

28. Valderas J, Alonso J (2008) Patient reported outcome measures: a model-based classification system for research and clinical practice. Qual Life Res 17:1125-1135

29. Valderas JM, Kotzeva A, Espallargues M et al. (2008) The impact of measuring patient-reported outcomes in clinical practice: a systematic review of the literature. Qual Life Res 17:179-193

30. Van Oppen JD, Keillor L, Mitchell Á et al. (2019) What older people want from emergency care: a systematic review. Emerg Med J 36:754-761 